Journal of Case Reports 2020;10(2):75-77

\title{
Diffuse Genital Herpes
}

\author{
Pratishtha Upadhyay, Yuye Li \\ Department of Dermatology and Venereology, Kunming Medical University First Affiliated Hospital, Yunnan, China.
}

\section{Corresponding Author: \\ Prof. Yuye Li \\ Email: yyeli2000@126.com}

This is an Open Access article distributed under the terms of the Creative Commons Attribution License (creativecommons.org/ licenses/by/3.0).

Received : January 3, 2020

Accepted : April 2,2020

Published : April 15,2020

\begin{abstract}
Background: Systemic lupus erythematosus is an autoimmune disease and genital herpes is a viral disease which can be aggravated during immunocompromised state. Case Report: A 45 years old female presented with rash in her buttocks for five years. She was a known case of SLE and was under immunosuppressive treatment for six years. The lesion on her buttocks were associated with mild pain but without itching. Lesions were erythematous with blisters and scab formation which were previously diagnosed at local hospital as erythematosus pemphigus. Histopathology and HSV -2 DNA testing by immunofluorescent method confirmed the diagnosis. Conclusion: This case illustrates diagnosis of atypical viral presentations in immunosuppressive people can be challenging. Viral antibody titers can give false negative results in immunocompromised patients.
\end{abstract}

Keywords: Blister, Buttocks, DNA, Herpes Genitals, Systemic Lupus Erythematosus, Pruritis.

\section{Introduction}

Systemic lupus erythematosus (SLE) is an autoimmune disease, can cause various comorbidities resulting in impaired quality of life. Herpes simplex virus (HSV) is a virus which stays in the body and recurring in immunocompromised host. The common site of HSV is oral and genital mucocutaneous areas but it can also be seen in ocular, pulmonary, gastrointestinal, hepatic, genitourinary and central nervous system and if not diagnosed and treated timely can lead to mortality [1]. Erythematosus pemphigus is a type of pemphigus which has the immunological features of both lupus erythematosus and pemphigus foliaceus. It has erythematous, scaly and crusted plaques in the nose and malar areas which also shows like butterfly distribution [2].

Here in we report a middle-aged female patient with SLE who presented with erythema and vesicles in her buttock region, her HSV-IgM was negative but viral load and histopathological test confirmed HSV-2 infection.

\section{Case Report}

A 45 years old female presented with a chief complaint of erythema and vesicles on the buttocks for 5 years. The lesions were not pruritic but mild pain was present. The problems used to aggravate during cold weather. The patient was previously diagnosed as a case of systemic lupus erythematosus 6 years back and two months back she was admitted to our hospital with complaints of abdominal pain and vomiting. She had undergone CT scan which showed pyloric gastric wall diffuse thickening edema, bilateral urethral dilatation, pelvic effusion which were suggestive of SLE. Then, she was confirmed as a case of SLE because of presence of the factors like anemia, leukocytopenia, anti-nuclear antibodies and antids DNA positive, proteinuria, positive rheumatoid factor, hypocomplementemia. She was under maintenance dose of prednisolone. She was also under tab hydroxychloroquine and tab leflunomide for rheumatoid arthritis. But during discharge from our hospital she was in the tapering dose of prednisolone. 
In the past 5 years, she had on and off lesions in the buttocks. The severity has now progressed to ulceration and scab formations. Erythematous pemphigus was diagnosed on lesion histopathology outside. During her visit to our outpatient department, she was well oriented with mild pallor and stable vital signs. On clinical examination of the lesion, small patchy erythematous lesions were present along with blisters, ulcerations and scabs on the skin of the buttocks were found [Fig. 1]. Repeat histopathological examination of the lesion showed superficial erosion with scab formation, residual epithelial cell lysis, some cell had chromatin edge collection, nuclear enlargement, lymphocyte and plasma cell infiltration in superficial and middle dermis which along with the clinical features were suggestive of herpes simplex or the herpes zoster. Along with histopathology patient was made to rule out other STDs like syphilis and HIV.

On the hematological examination, she was negative for syphilis and HIV, IgG positive for both HSV-1/ HSV-2 and IgM was negative for both. HSV DNA test was positive for HSV-2 whereas HSV-1 was within the normal limit. Her final diagnosis was HSV-2 infection with SLE. After the confirmation of the HSV infection in the patient, she was started treatment with valacyclovir orally and penciclovir ointment along with the immune modulators. After initiation of the antiviral drug therapy and immunomodulators for 2 months, erythema, vesicles and rashes were cured with no recurrence, and she is currently being followed up and treated for SLE in the rheumatology department.

\section{Discussion}

SLE is a chronic autoimmune disease which is characterized by the presence of auto-antibodies against nuclear antigen, immune complex deposition and tissue damage in skin, kidney, heart and lungs [3]. Pemphigus is also an auto immune disease which has clinical manifestation of intra-epidermal blistering of the skin and/or

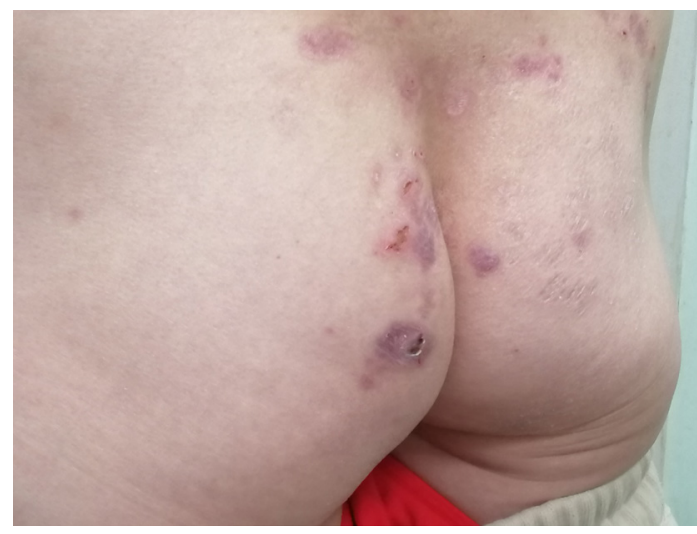

Fig.1: Erythema, blisters and scab lesions in buttock area.
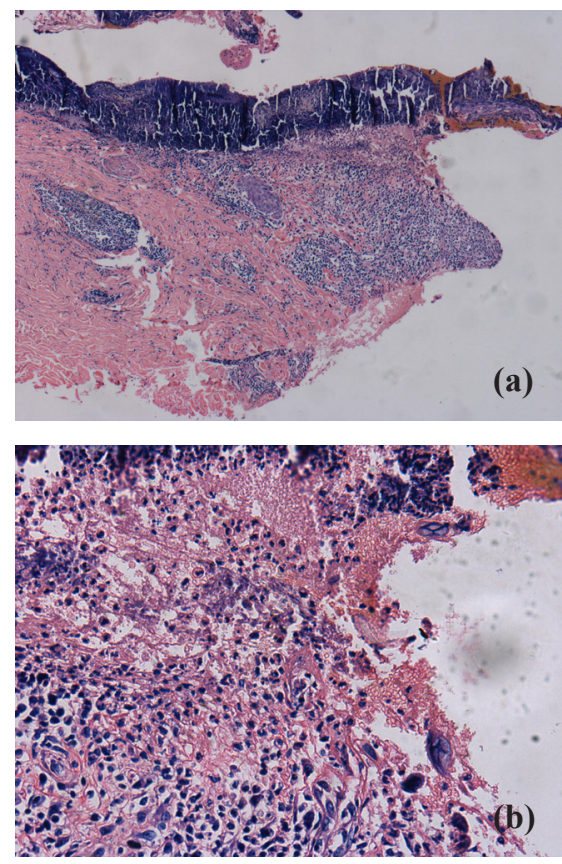

Fig.2 (a,b): Histopathology of the rash on buttock area: Haematoxylin and Eosin (H\&E 2a: $x 100$ and 2b: x400 magnification) showed superficial erosion with scab formation, residual epithelial cell lysis, chromatin edge collection, enlarged nucleus, lymphocyte and plasma cell infiltration in superficial and middle dermis.

mucus membrane [4]. The relationship among lupus erythematosus and pemphigus is a longdebated topic [5]. Both SLE and pemphigus are B cell mediated auto-immune disease. The pathogenesis of auto-immune disease still remains unknown. Histopathologically, they seem to have same acantholysis in the epidermis and eroded 
and crusted lesions are present. According to the European league against rheumatism (EULAR) every SLE patient must undergo test for various viral test before starting the treatment [6].

On the other side genital herpes is an incurable, chronic disease which shows lesion like blister, erosion as early manifestation and scabies as late manifestation and is painful and recurrent. Virus being in the dormant phase gets activated in immunocompromised state $[7,8]$. Acute viral infection can mimic SLE, aggravate SLE and lupus flares in the already diagnosed SLE patient [10]. This case was initially diagnosed with erythematous pemphigus. Her initial antibody titers failed to detect HSV-1 and HSV-2 may be because of window period. Histopathological examination showed etiology as herpes virus. Viral loads for HSV-2 was positive. Recently, on the cohort study done in Taiwan showed that patient diagnosed with SLE are at higher risk of HSV infection in view of risk factors like age more than 18 years, mucocutaenous infection and steroid therapy [1].

High concentration of antibody against viruses like HSV-1, HSV-2, EBV and CMV are seen in SLE patients as compared with controls [11]. The patient diagnosed with SLE are more predisposed to develop HSV esophagitis and enteritis [12]. In our case also, the patient also had similar history in the past but the patient was treated in the line of SLE only and HSV infection was not even considered initially. The patient was being treated for SLE with steroid therapy which might be a cause for immunosuppression. Her immunosuppressed status was risk factor for HSV infection. But during initial presentation to the hospital, patient conditions were thought to be consequences of SLE but not HSV, which might be the reason for its misdiagnosis.

\section{Conclusion}

SLE patients require high index of suspicion for diagnosis of opportunistic infections like HSV infection.
Contributors: PU: manuscript writing, literature review and data acquisition; YL: manuscript editing, and critical inputs into the manuscript. YL will act as a study guarantor. Both authors approved the final version of this manuscript and are responsible for all aspects of the study.

Funding: None; Competing interests: None stated.

\section{References}

1. Li TH, Lai CC, Wang WH, Chan WS, Tsao YP, Tsai CY, et al. Risk of severe herpes simplex virus infection in systemic lupus erythematosus: Analysis of epidemiology and risk factors analysis in Taiwan. Ann Rheum Dis. 2019;78:941-946.

2. Weedon D. The vesiculobullous reaction pattern. In: Weedon's Skin Pathology. 54 ${ }^{\text {th }}$ ed. 2010;123-168.

3. Herrada AA, Escobedo N, Iruretagoyena M, Valenzuela RA, Burgos PI, Cuitino L, et al. Innate immune cells' contribution to systemic lupus erythematosus. Front Immunol. 2019;10:772.

4. Oktarina DAM, Poot AM, Kramer D, Diercks GFH, Jonkman MF, Pas HH. The IgG "lupus-band" deposition pattern of pemphigus erythematosus: Association with the desmoglein 1 ectodomain as revealed by 3 cases. Arch Dermatol. 2012;148:1173-1178.

5. Chavan SA, Sharma YK, Deo K, Buch AC. A case of senear-usher syndrome. Indian J Dermatol. 2013;58:329.

6. Sebastiani GD, Iuliano A, Prevete I, Minisola G. Opportunistic infections in systemic lupus erythematosus. Int J Clin Rheumtol. 2012;7:275-279.

7. Sezin T, Vorobyev A, Sadik CD, Zillikens D, Gupta Y, Ludwig RJ. Gene expression analysis reveals novel shared gene signatures and candidate molecular mechanisms between pemphigus and systemic lupus erythematosus in CD4+ T cells. Front Immunol. 2018;8:1992.

8. Cojocaru M, Cojocaru IM, Silosi I. Multiple autoimmune syndrome. Maedica (Buchar). 2010;5:132-134.

9. Sawamura S, Kajihara I, Makino K, Makino T, Fukushima $\mathrm{S}$, Jinnin $\mathrm{M}$, et al. Systemic lupus erythematosus associated with myasthenia gravis, pemphigus foliaceus and chronic thyroiditis after thymectomy. Australas J Dermatol. 2017;58:e120-e122.

10. Theofilopoulos AN, Baccala R, Beutler B, Kono DH. Type I interferons (A/B) in immunity and autoimmunity. Annu Rev Immunol. 2005;23:307-335.

11. Shin H, Iwasaki A. Generating protective immunity against genital herpes. Trends Immunol. 2013;34:487-494.

12. Vista ES, Weisman MH, Ishimori ML, Chen H, Bourn RL, Bruner BF, et al. Strong viral associations with SLE among Filipinos. Lupus Sci Med. 2017;4:e000214.

13. Wakui H, Togashi M, Omokawa A, Okuyama S, Masai R, Komatsuda A, et al. Simultaneous herpes simplex virus esophagitis and lupus enteritis in a patient with systemic lupus erythematosus. Mod Rheumatol. 2010;20:98-101. 